\title{
Percutaneous radiofrequency ablation of primary intraosseous spinal glomus tumor
}

\author{
Fabio Becce • Delphine Richarme • Igor Letovanec • \\ Willy Gilgien • Nicolas Theumann
}

Received: 14 May 2011 /Revised: 3 October 2011 / Accepted: 6 October 2011 / Published online: 10 November 2011

(C) ISS 2011

\begin{abstract}
The glomus tumor is a rare, benign, but painful vascular neoplasm arising from the neuromyoarterial glomus. Primary intraosseous glomus tumor is even rarer, with only about 20 cases reported in the literature so far, 5 of which involved the spine. Surgical resection is currently considered the treatment of choice. We herewith present an uncommon case of primary intraosseous spinal glomus tumor involving the right pedicle of the eleventh thoracic vertebra (T11). To our knowledge, this is the first case of primary intraosseous spinal glomus tumor successfully treated by percutaneous CT-guided radiofrequency ablation (RFA).
\end{abstract}

Keywords Radiofrequency ablation - Glomus tumor . Intraosseous tumor Thoracic spine · Imaging

\section{Introduction}

The glomus tumor is a rare, benign, but painful vascular neoplasm derived from the neuromyoarterial glomus and

\footnotetext{
F. Becce $(\square) \cdot$ D. Richarme $\cdot$ N. Theumann

Rue du Bugnon 46,

1011 Lausanne, Switzerland

e-mail: fabio.becce@chuv.ch

I. Letovanec

University Institute of Pathology,

Centre Hospitalier Universitaire Vaudois,

1011 Lausanne, Switzerland

\section{W. Gilgien}

University Institute of General Medicine,

Policlinique Médicale Universitaire,

1011 Lausanne, Switzerland
}

Department of Diagnostic and Interventional Radiology,

Centre Hospitalier Universitaire Vaudois, University of Lausanne, accounts for approximately $2 \%$ of all soft tissue tumors [1, 2]. Patients are usually affected in the third to fifth decades of life, with no gender predominance. This tumor primarily occurs in the distal extremities, especially the subungual regions. Primary intraosseous glomus tumor is extremely rare, with only about 20 cases reported in the literature so far [3]. Five of those cases involved the spinal axis (Table 1) [4-8]. To date, complete surgical excision is considered the treatment of choice [8].

We herewith present an uncommon case of primary intraosseous spinal glomus tumor involving the right pedicle of the eleventh thoracic vertebra (T11). To our knowledge, this is only the sixth case of primary intraosseous spinal glomus tumor reported and the first to be treated by percutaneous CT-guided radiofrequency ablation (RFA).

\section{Case report}

A 73-year-old woman was referred to our hospital for evaluation and management of a focal vertebral lesion. She presented with a 6-month history of upper and lower back pain, which persisted despite the administration of steroidal and non-steroidal anti-inflammatory drugs. The pain was centered on the thoraco-lumbar junction and radiated down to the lower back and, at times, the right leg. There was no history of trauma, osteoporosis or cancer.

On physical examination, the patient had a good range of motion in the thoracic and lumbar spine. There was no paraspinal muscle spasm, but pain on deep palpation at the thoraco-lumbar junction. The straight leg raise test and motor and sensory examinations were all normal.

All imaging studies were performed in another institution. Anteroposterior and lateral radiographs of the thoracic 


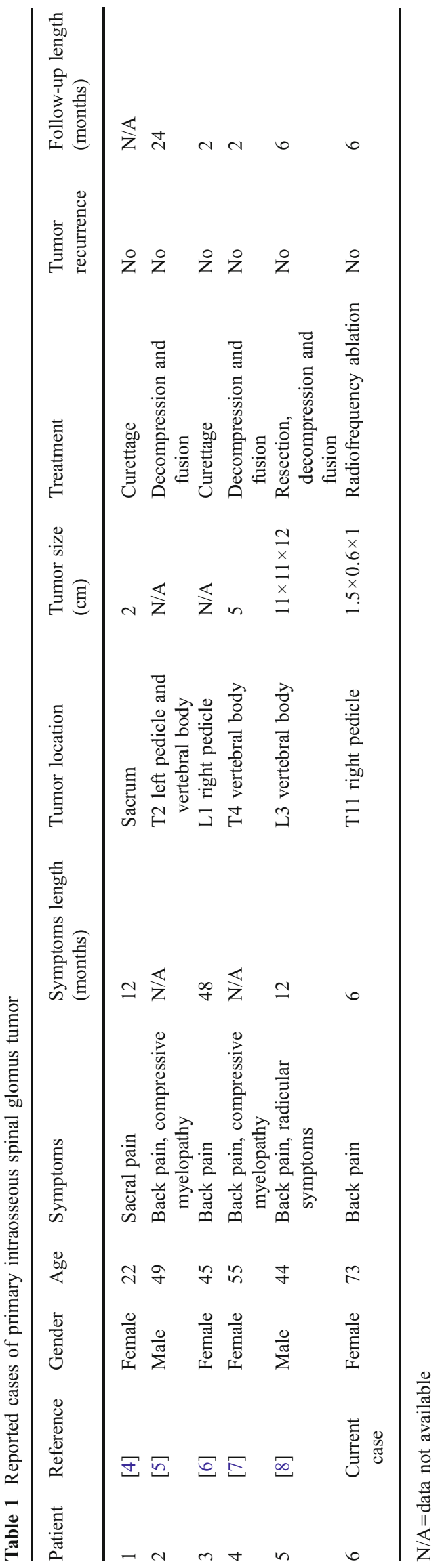

and lumbar spine showed only mild degenerative spur formation in the lumbar spine. On multidetector computed tomography (CT), a well-circumscribed, lobulated osteolytic lesion was noted in the right pedicle of T11 (Fig. 1). It measured $1.5 \times 0.6 \mathrm{~cm}$ in the transverse plane and $1 \mathrm{~cm}$ in its cranio-caudal axis. This lesion was surrounded by peripheral osteosclerosis. There was no periosteal reaction or cortical bone discontinuity. Magnetic resonance (MR) imaging of the thoracic spine demonstrated a relatively homogeneous oblong lesion with low signal intensity on T1-weighted (Fig. 2a) and high signal intensity on T2-weighted images (Fig. 2b), which was avidly enhanced after gadolinium chelate administration (Fig. 2c, d). There was minimal peripheral bone marrow edema. No extraosseous component was observed. In retrospect, this lesion was already present on a thoracic CT angiography performed 1 year earlier, and had slightly increased in size since then. A percutaneous CT-guided core biopsy of the right pedicle of T11 was then achieved under local anesthesia (rapidocain 2\%; Sintetica, Mendrisio, Switzerland) using an 11-gauge coaxial bone biopsy system (Somatex Safe-Cut; Somatex Medical Technologies, Teltow, Germany). Surprisingly, this procedure was well tolerated by the patient, triggering only mild pain when the cannula went through the lesion. Histopathological examination revealed a diagnosis of primary intraosseous glomus tumor, with intraosseous sheets of medium-sized bland cells (Fig. 3a, b) that stained strongly for smooth muscle actin at immunohistochemistry (Fig. 3c). No mitotic activity was observed. According to the criteria proposed by Folpe et al. [9], the tumor was classified as a "glomus tumor of uncertain malignant potential" owing to its deep location only.

Given that the patient refused surgical intervention, but still presented disabling symptoms, a minimally invasive, percutaneous CT-guided RFA was scheduled under general anesthesia (Fig. 4), after obtaining the patient's written informed consent. A single dose (1 g) of intravenous cefazolin was administered to the patient $30 \mathrm{~min}$ before the procedure. After adequate grounding and under strict aseptic conditions, access to the lesion was gained using a 14-gauge coaxial drill system (Bonopty Penetration Set; Radi Medical Systems, Uppsala, Sweden) through a dorsal approach and under CT guidance. The inner needle was then replaced by a 20-gauge radiofrequency (RF) cannula bearing a 5-mm active tip (RFK; Cosman Medical, Burlington, MA, USA). After controlling the accurate position of the RF probe tip by CT fluoroscopy (SmartStep; GE Healthcare, Waukesha, WI, USA), the electrode was connected to the RF generator (RFG-3 C; Radionics, Burlington, MA, USA) and the procedure achieved by maintaining the tip temperature to $80^{\circ} \mathrm{C}$ for two cycles of 3 min each, with a 2 -minute free interval. The RF probe 

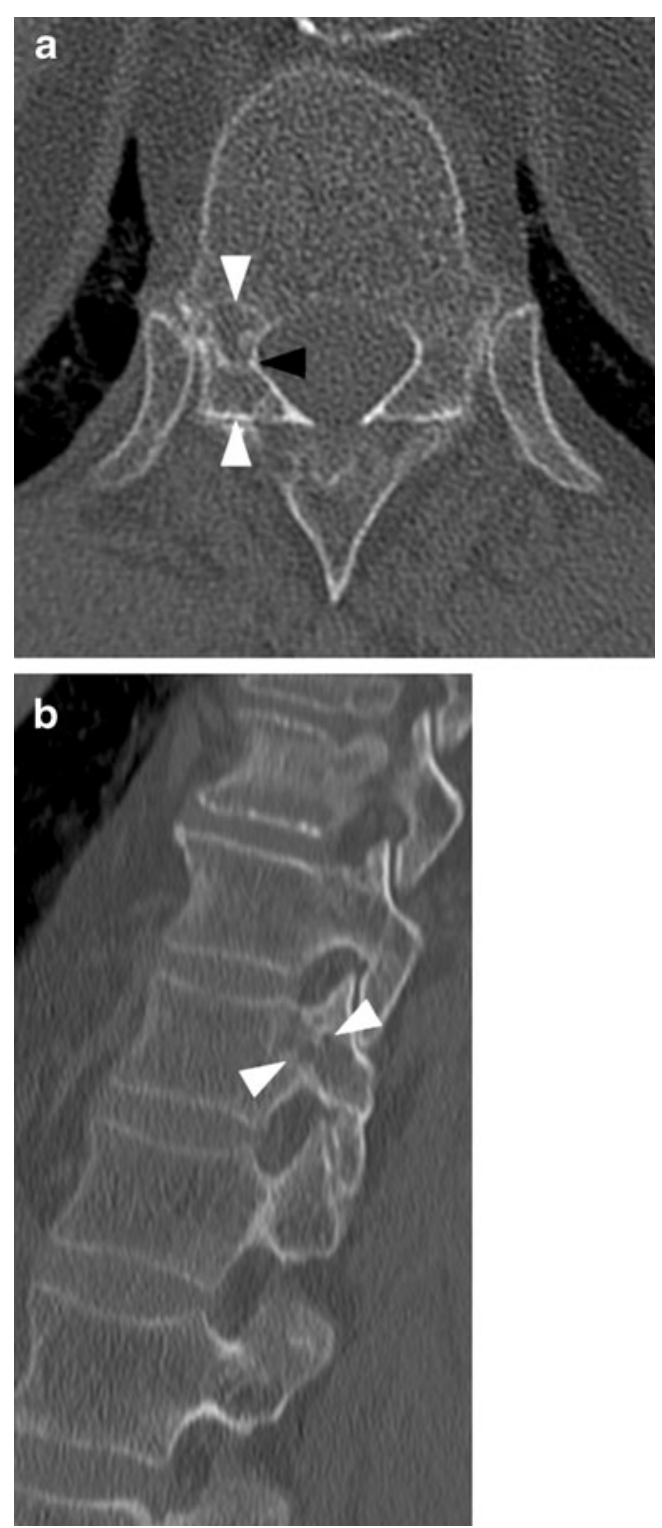

Fig. 1 a Axial and b sagittal-reformatted unenhanced CT images of the thoraco-lumbar spine show a well-circumscribed, lobulated osteolytic lesion in the right pedicle of the eleventh thoracic vertebra (T11), surrounded by peripheral osteosclerosis (white arrowheads). There is no periosteal reaction. The cortical margins of the pedicle are preserved (black arrowhead)

was then pulled approximately $8 \mathrm{~mm}$ backward under CT fluoroscopy and a second contiguous RFA treatment was performed within the posterior portion of the lesion.

One day after the RFA procedure, the patient left the hospital symptom-free. At the 3-month follow-up examination, she presented again with lower back pain, which was due to L5-S1 degenerative disc disease with Modic type 1 vertebral bone marrow changes on MR imaging. However, the previous symptoms could not be reproduced on deep palpation at the thoraco-lumbar junction. A second follow-up MR imaging of the thoraco-lumbar spine obtained 6 months after the RFA revealed minor residual peripheral bone marrow edema, but no features suggestive of residual or recurrent disease.

\section{Discussion}

Unlike paragangliomas, which originate from chromaffin cells, glomus tumors (also called angioglomoid tumors) are benign vascular neoplasms arising from smooth muscle cells of the neuromyoarterial glomus [1, 10]. Normal glomus bodies are specialized complexes of vascular anastomoses that operate as regulators of body temperature. These are mainly located in the subcutaneous tissue of the extremities, especially the palmar and plantar areas and the subungual regions $[1,10]$. However, a few cases of glomus tumor affecting the stomach, the chest wall, the mediastinum, the penis, and the face have been reported $[1,10]$. Intraosseous glomus tumors are uncommon and may be related to secondary involvement of a soft tissue lesion. Iglesias de la Torre et al. were the first to report a case in 1939 [11]. Since then, only about 20 additional cases of intraosseous glomus tumor have been published $[3,12,13]$. Most of those involved the phalanges of the fingers (12 out of $21,57 \%)[12,13]$ and 5 affected the spinal axis (5 out of 21, 24\%; Table 1) [4-8]. The patients' median age was 36 years (range 22-68 years) and a female predominance (13 out of 19,68\%) was noted [3].

Excluding epithelial tumors, the incidence of glomus tumor was $1.6 \%$ of 500 consecutive soft tissue tumors in the 1955 Mayo Clinic series [2]. Despite its rarity, this neoplasm is relatively well-known owing to its typical clinical features. The clinical triad of excruciating pain, exquisite point tenderness, and cold sensitivity is observed in approximately $30 \%$ of cases [14]. However, due to its small size, the tumor is often not visible or palpable on physical examination [1]. In the spine, back pain is the most common symptom, usually lancinating and sharp, and may be complicated by neurological compression symptoms (Table 1) [4-8]. No patient with a spinal glomus tumor has yet presented with the clinical triad (Table 1). In our case, the patient's radicular symptoms were probably due to concomitant L5-S1 degenerative disc disease, but not related to the glomus tumor itself.

The roentgenological and MR imaging features of extraosseous glomus tumor are well recognized [14, 15]. Conventional radiographs are frequently normal, but a small scalloped bony defect in a distal phalanx may be seen [15]. On MR imaging, the tumor is typically of high signal intensity on $\mathrm{T} 2$-weighted and low signal intensity on T1-weighted images, with strong enhancement after intravenous gadolinium administration [14]. To our knowledge, there are no case series reporting the imaging characteristics of intraosseous glomus tumor. In our case, the CT and MR imaging findings were similar to those in two of the 
Fig. 2 a Sagittal T1-weighted fast spin echo (FSE; TR/TE $=$ 400/9), b fat-suppressed T2weighted FSE $(3,200 / 80)$, and c sagittal and $\mathbf{d}$ axial gadoliniumenhanced fat-suppressed T1weighted FSE (425/9) MR images of the thoraco-lumbar spine display a relatively homogeneous oblong lesion involving the right pedicle of the eleventh thoracic vertebra (T11). The lesion is of low signal intensity on T1-weighted (a, black arrowheads) and high signal intensity on T2-weighted (b, white arrowheads) images. It is avidly enhanced after gadolinium chelate administration (c, $\mathbf{d}$, arrow). The lesion has no extraosseous component
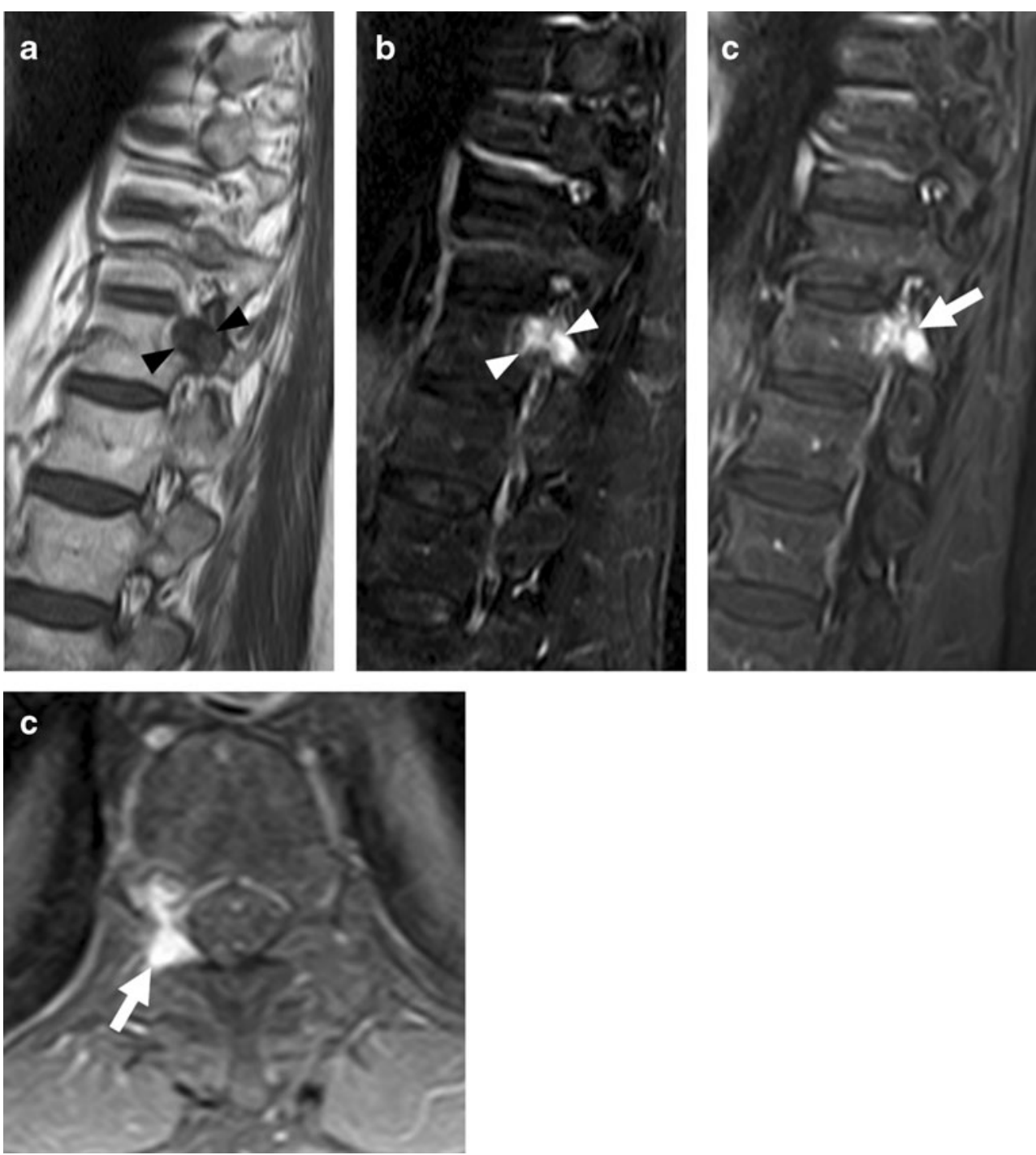

previously reported cases $[6,8]$. Owing to the rarity and non-specific imaging features of this tumor in the spine, the diagnosis of intraosseous glomus tumor was not considered prior to core needle biopsy. The main differential diagnosis consisted of metastasis, plasmacytoma, lymphoma, giant cell tumor or osteoid osteoma [16].

Complete surgical excision by curettage or en bloc resection is currently considered to be the treatment of choice [4-8]. In the five previously reported cases of intraosseous spinal glomus tumor, no recurrence was observed following surgery, with a mean follow-up time of 8 months (range 2-24 months; Table 1). To our knowledge, this is the first case of primary intraosseous spinal glomus tumor to be treated by percutaneous CTguided RFA. Since the first experiments by Tillotson et al. in 1989 [17], RFA has now become recognized to be at least as effective as surgery in the treatment of osteoid osteoma, with similar success rates, but shorter hospitalization length and fewer complications [18]. Subsequently,
RFA was also successfully applied in the treatment of chondroblastoma [19, 20], eosinophilic granuloma [20, 21], bone metastases [22], and even Brodie's abscesses [20]. However, percutaneous ablation of spinal bone tumors is still relatively limited by the fear of thermal injury to the adjacent neurological structures [23]. Although Dupuy et al. reported decreased heat transmission in cancellous bone and insulative effect of cortical bone [24], cytotoxicity to the spinal cord may appear at temperatures higher than $45^{\circ} \mathrm{C}$. In our case, given the intact cortical margins of the right pedicle of T11, we opted not to employ additional protective measures advocated in prior publications, such as epidural instillation of cooled fluids [23], although the nearest neural structures were $4 \mathrm{~mm}$ away in the transverse and $6 \mathrm{~mm}$ away in the coronal plane. Following RFA, the patient reported immediate pain relief and no symptoms related to radicular or spinal cord injury was noted. Moreover, no signal abnormalities of the neural structures were found on follow-up MR imaging studies. 

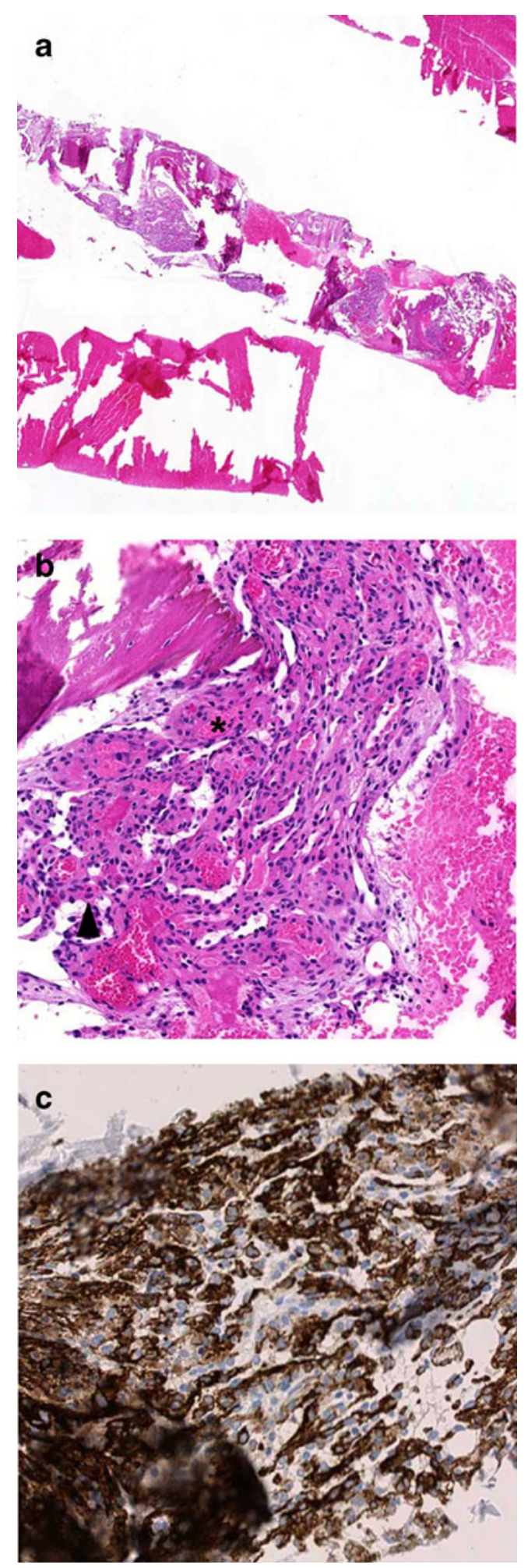

Although Rosenthal et al. first demonstrated that single $\mathrm{RF}$ cycles of 3 to 4 min would be effective to treat most osteoid osteomas [18], some authors now prefer $6 \mathrm{~min}$ ablations at $90^{\circ} \mathrm{C}$ because they noted lower recurrence rates $[25,26]$. In our experience, we are currently applying two

Fig. 3 Photomicrographs of the core needle biopsy specimen. a Core bone biopsy reveals intraosseous sheets of medium-sized bland cells with vessels of variable size (H\&E stain; original magnification, $\times 10)$. b Glomus cells (arrowhead) are round to oval and display round nuclei, amphophilic cytoplasm and no atypia. The adjacent vessels (asterisk) are of variable size (H\&E stain; original magnification, $\times 100$ ). c At immunohistochemistry, glomus cells are strongly and diffusely positive for smooth muscle actin (original magnification, $\times 200$ )

cycle RF treatments inspired by the technique used by Rimondi et al. $[25,26]$. Indeed, these authors propose to gradually raise the tip temperature to $60^{\circ} \mathrm{C}$ for $2 \mathrm{~min}$ to reduce tissue impedance and improve RF generator output, and then proceed to $14-15 \mathrm{~min}$ of ablation at $90-93^{\circ} \mathrm{C}$. In our particular case, we decided to reduce the temperature and length of the second RF cycle owing to the spinal location of the lesion and the proximity of the neural structures.

In conclusion, although the follow-up length is relatively short, this case illustrates a potential additional application of percutaneous image-guided thermal ablation of spinal bone tumors.

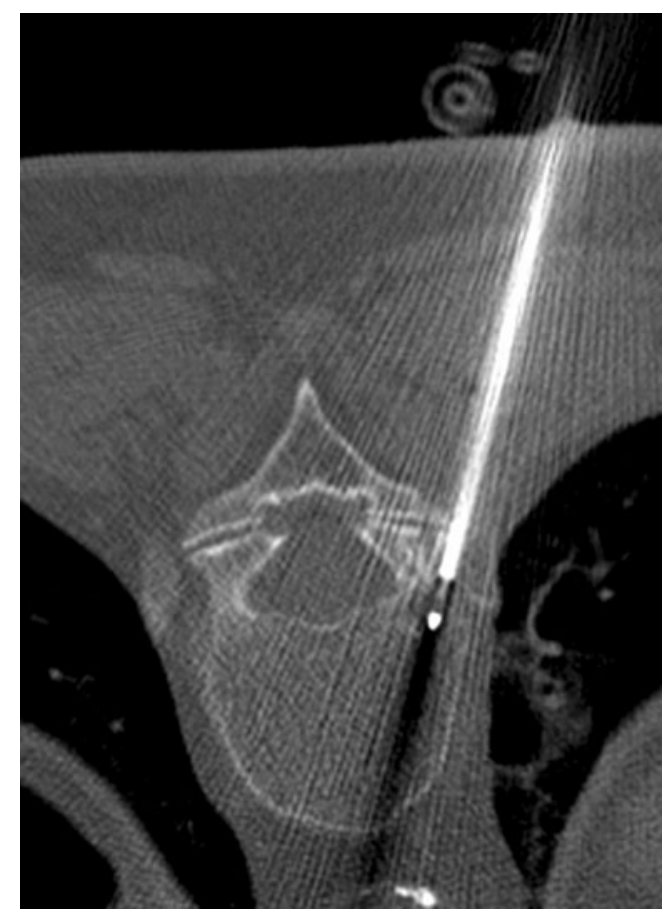

Fig. 4 Per-procedural axial unenhanced CT image at the level of the eleventh thoracic vertebra (T11) demonstrates the accurate position of the radiofrequency $(\mathrm{RF})$ probe tip within the anterior portion of the lesion of the T11 right pedicle. The outer cannula has not yet been withdrawn for treatment. The RF probe was subsequently pulled posteriorly to treat the posterior portion of the lesion with a second RFA. The nearest neural structures were distant $4 \mathrm{~mm}$ in the axial and $6 \mathrm{~mm}$ in the coronal plane 
Acknowledgements The authors gratefully thank Dr. Anne-Sophie Knoepfli for her assistance with manuscript editing.

Conflict of interest None.

\section{References}

1. Resnick D, Kyriakos M, Greenway GD. Tumors and tumor-like lesions of bone: imaging and pathology of specific lesions. In: Resnick D, editor. Diagnosis of bone and joint disorders. Philadelphia: Saunders; 2002. p. 3995-9.

2. Soule EH, Ghormley RK, Bulbulian AH. Primary tumors of the soft tissues of the extremities exclusive of epithelial tumors: an analysis of five hundred consecutive cases. AMA Arch Surg. 1955;70:462-74.

3. Urakawa H, Nakashima H, Yamada Y, Tsushima M, Ohta T, Nishio T. Intraosseous glomus tumor of the ulna: a case report with radiographic findings and review of the literature. Nagoya $\mathrm{J}$ Med Sci. 2008;70:127-33.

4. Kobayashi Y, Kawaguchi T, Imoto K, Yamamoto T. Intraosseous glomus tumor in the sacrum. A case report. Acta Pathol Jpn. 1990;40:856-9.

5. Bessho Y, Kataoka O, Sho T, Kitazawa S, Okada S. Intraosseous glomus tumor in the upper thoracic spine complicating compression myelopathy. A case report. Spine. 1991;16:988-90.

6. Robinson JC, Kilpatrick SE, Kelly Jr DL. Intraosseous glomus tumor of the spine. Case report and review of the literature. J Neurosurg. 1996;85:344-7.

7. Payer M, Grob D, Benini A, Varga S, Hodler J, Martin JB. Intraosseous glomus tumor of the thoracic spine. Case illustration. J Neurosurg. 2002;96:137.

8. Bambakidis NC, Gore P, Eschbacher J, Coons S, Albuquerque FC. Intraosseous spinal glomus tumors: case report. Neurosurgery. 2007;60:1152-3.

9. Folpe AL, Fanburg-Smith JC, Miettinen M, Weiss SW. Atypical and malignant glomus tumours: analysis of 52 cases, with a proposal for the reclassification of glomus tumours. Am J Surg Pathol. 2001;25:1-12.

10. Kransdorf MJ, Murphey MD. Vascular and lymphatic tumors. In: Kransdorf MJ, Murphy MD, editors. Imaging of soft tissue tumors. Philadelphia: Lippincott Williams Wilkins; 2006. p. $150-88$.

11. Iglesias de la Torre L, Gomez Camejo M, Palacios G. Consideraciones clinicas, radiologicas y quirurgicas del glomus tumoral de Masson. Cirurg Ortop Traumatol Habana. 1939;7:11-7.
12. Bjorkengren AG, Resnick D, Haghighi P, Sartoris DJ. Intraosseous glomus tumor: report of a case and review of the literature. Am J Roentgenol. 1986;147:739-41.

13. Simmons TJ, Bassler TJ, Schwinn CP, Forrester DM. Case report 749. Primary glomus tumor of bone. Skeletal Radiol. 1992;21:407-9.

14. Drapé JL, Idy-Peretti I, Goettmann S, et al. Subungual glomus tumors: evaluation with MR imaging. Radiology. 1995;195:507-15.

15. Mathis Jr WH, Schulz MD. Roentgen diagnosis of glomus tumors. Radiology. 1948;51:71-6.

16. Murphey MD, Andrews CL, Flemming DJ, Temple HT, Smith WS, Smirniotopoulos JG. From the archives of the AFIP. Primary tumors of the spine: radiologic-pathologic correlation. Radiographics. 1996;16:1131-58.

17. Tillotson CL, Rosenberg AE, Rosenthal DI. Controlled thermal injury of bone. Report of a percutaneous technique using radiofrequency electrode and generator. Invest Radiol. 1989;24:888-92.

18. Rosenthal DI, Hornicek FJ, Wolfe MW, Jennings LC, Gebhardt MC, Mankin HJ. Percutaneous radiofrequency coagulation of osteoid osteoma compared with operative treatment. J Bone Joint Surg Am. 1998;80:815-21.

19. Rybak LD, Rosenthal DI, Wittig JC. Chondroblastoma: radiofrequency ablation - alternative to surgical resection in selected cases. Radiology. 2009;251:599-604.

20. Becce F, Theumann N, Rochette A, et al. Osteoid osteoma and osteoid osteoma-mimicking lesions: biopsy findings, distinctive MDCT features and treatment by radiofrequency ablation. Eur Radiol. 2010;20:2439-46.

21. Corby RR, Stacy GS, Peabody TD, Dixon LB. Radiofrequency ablation of solitary eosinophilic granuloma of bone. Am J Roentgenol. 2008;190:1492-4.

22. Callstrom MR, Charboneau JW, Goetz MP, et al. Painful metastases involving bone: feasibility of percutaneous CT- and US-guided radio-frequency ablation. Radiology. 2002;224:87-97.

23. Rybak LD, Gangi A, Buy X, La Rocca Vieira R, Wittig J. Thermal ablation of spinal osteoid osteomas close to neural elements: technical considerations. Am J Roentgenol. 2010;195: W293-298.

24. Dupuy DE, Hong R, Oliver B, Goldberg SN. Radiofrequency ablation of spinal tumors: temperature distribution in the spinal canal. Am J Roentgenol. 2000;175:1263-6.

25. Rimondi E, Bianchi G, Malaguti MC, et al. Radiofrequency thermoablation of primary non-spinal osteoid osteoma: optimization of the procedure. Eur Radiol. 2005;15:1393-9.

26. Rimondi E, Mavrogenis AF, Rossi G, et al. Radiofrequency ablation for non-spinal osteoid osteomas in 557 patients. Eur Radiol. 2011; doi:10.1007/s00330-011-2240-1 